\title{
CARDINAL HERMITE SPLINE INTERPOLATION WITH SHIFTED NODES
}

\author{
GERLIND PLONKA AND MANFRED TASCHE
}

\begin{abstract}
Generalized cardinal Hermite spline interpolation is considered. A special case of this problem is the classical cardinal Hermite spline interpolation with shifted nodes. By means of a corresponding symbol new representations of the cardinal Hermite fundamental splines can be given. Furthermore, a new efficient algorithm for the computation of the cardinal Hermite spline interpolant is obtained, which is mainly based on fast Fourier transform. This algorithm is shown to be also applicable to computing the periodic Hermite spline interpolant. In both cases we only use necessary and sufficient conditions for the existence and uniqueness of the corresponding Hermite spline interpolant.
\end{abstract}

\section{INTRODUCTION}

In this paper we shall construct new efficient algorithms for the computation of the Hermite fundamental splines and the Hermite spline interpolant.

We use standard notation: Let $f^{\wedge}$ be the Fourier transform

$$
f^{\wedge}(u):=\int_{-\infty}^{\infty} f(x) e^{-i u x} d x \quad(u \in \mathbb{R})
$$

of $f \in L_{1}(\mathbb{R})$. Assume that

$$
\sum_{j=-\infty}^{\infty} f^{\wedge}(u+2 \pi j) \quad(u \in \mathbb{R})
$$

is uniformly convergent on $\mathbb{R}$. Then $f^{\sim}$ with

$$
f^{\sim}(u):=\sum_{j=-\infty}^{\infty} f^{\wedge}(u+2 \pi j) \quad(u \in \mathbb{R})
$$

is called the $2 \pi$-periodization of $f^{\wedge}$. The Poisson summation formula reads as follows:

$$
f^{\sim}(u)=\sum_{j=-\infty}^{\infty} f(j) e^{-i u j}
$$

Consider equidistant knots with multiplicity 2 ,

$$
x_{j}:=\lfloor j / 2\rfloor \quad(j \in \mathbb{Z}),
$$

Received by the editor December 16, 1992 and, in revised form, August 20, 1993.

1991 Mathematics Subject Classification. Primary 65D05, 65D07, 65T10.

Key words and phrases. Cardinal Hermite spline interpolation, fast Fourier transform, generalized Euler-Frobenius polynomials. 
where $\lfloor j / 2\rfloor$ denotes the integer part of $j / 2$. With $N_{m, \nu}^{2} \in C^{m-2}(\mathbb{R}) \quad(\nu=$ $0,1)$ we denote the normalized B-splines of degree $m(m \geq 2)$ and defect 2 with knots $x_{\nu}, x_{\nu+1}, \ldots, x_{\nu+m+1}$.

Let $S_{m, 2}^{0}(\mathbb{Z})$ be the set of all finite linear combinations of shifts of $N_{m, \nu}^{2}$ $(\nu=0,1)$ :

$$
\sum_{j=-\infty}^{\infty}\left(a_{j} N_{m, 0}^{2}(x-j)+b_{j} N_{m, 1}^{2}(x-j)\right) \quad\left(x \in \mathbb{R} ; a_{j}, b_{j} \in \mathbb{R}\right) .
$$

The $L_{1}(\mathbb{R})$-closure of $S_{m, 2}^{0}(\mathbb{Z})$ is denoted by $S_{m, 2}(\mathbb{Z})$. We consider the following generalized cardinal Hermite spline interpolation problem: For given data sequences $\mathbf{y}^{(0)}:=\left\{y_{j}^{(0)}\right\}_{j=-\infty}^{\infty}, \mathbf{y}^{(1)}:=\left\{y_{j}^{(1)}\right\}_{j=-\infty}^{\infty} \in l_{1}$, and shift parameters $\tau_{0}, \tau_{1} \in \mathbb{R}$ with $0<\tau_{0} \leq \tau_{1} \leq 1$, find a spline function $s \in S_{m, 2}(\mathbb{Z})$ such that

$$
s\left(k+\tau_{0}\right)=y_{k}^{(0)}, \quad\left[s(k+\cdot): \tau_{0}, \tau_{1}\right]=y_{k}^{(1)} \quad(k \in \mathbb{Z}),
$$

where $\left[s(k+\cdot): \tau_{0}, \tau_{1}\right]$ denotes the divided difference. In the case $\tau_{0}=\tau_{1} \in$ $(0,1]$ we obtain the classical cardinal Hermite spline interpolation problem.

For the sake of simplicity, we describe our method only for splines of defect 2. But the procedure can be simply generalized to the corresponding Hermite interpolation problem for splines with higher defect. Further, the Hermite spline interpolation problem (1) is of special interest in applications. The existence and uniqueness problem is generally solved only for defect 2 (cf. [5]).

In the literature, cardinal Hermite spline interpolation problems have been investigated mainly in the special case of nonshifted nodes (cf. $[2,10,1])$. In particular, the construction of fundamental splines and the existence and uniqueness of solutions have been studied. The periodic Hermite spline interpolation for defect $r>1 \quad(r \in \mathbb{N})$ with interpolation nodes $\tau_{0}=\tau_{1}=\cdots=$ $\tau_{r-1}=1$ have been treated in [3] and [4]. The more general periodic case $\tau_{0}=\tau_{1}=\cdots=\tau_{r-1} \in(0,1]$ can be found in [9].

Contrary to most of the other papers dealing with periodic or cardinal Hermite spline interpolation (cf. $[8,9,3,4]$ ) we do not use the normal B-splines $N_{m}(\cdot-k) \quad(k \in \mathbb{Z})$ and their derivatives (or their periodizations) as a basis for the spline space with higher defect, but instead B-splines with multiple knots.

In $\S 2$, we introduce a new representation of the symbol of the problem considered. This symbol will be the main tool of our approach to the cardinal fundamental splines in $\S 3$. Using fast Fourier transform, we obtain a new efficient algorithm for the computation of the cardinal Hermite spline interpolant in $\S 4$. Further, the close connection between periodic and cardinal Hermite spline interpolation will be considered. In $\S 5$, it will be shown that one and the same algorithm can be applied to the computation of the cardinal and the periodic spline interpolants.

\section{Symbol of CARDinal Hermite SPLINE INTERPOlation}

Similar to Lagrange spline interpolation, we have to investigate the symbol of the interpolation problem (1). First we introduce the Euler-Frobenius polynomials of multiplicity 2 in order to construct the symbol for the generalized Hermite spline interpolation problem (1). 
With the help of B-splines with double knots, the generalized Euler-Frobenius polynomials of multiplicity 2 with shift parameter $x \in \mathbb{R}$ can be defined by

$$
H_{m, \nu}^{2}(x, z):=\sum_{j=-\infty}^{\infty} N_{m, \nu}^{2}(j+x) z^{j} \quad(\nu=0,1 ; z \in \mathbb{C}) .
$$

Since $\operatorname{supp} N_{m, \nu}^{2} \quad(\nu=0,1)$ is compact, the functions $H_{m, \nu}^{2} \quad(\nu=0,1)$ are well defined. We are especially interested in the behavior of $H_{m, \nu}^{2}(\nu=0,1)$ on the unit circle. Therefore, we put for $u \in \mathbb{R}$

$$
h_{m, \nu}^{2}(x, u):=H_{m, \nu}^{2}\left(x, e^{-i u}\right) \quad(x \in \mathbb{R}) .
$$

Then we obtain the following properties of $h_{m, \nu}^{2}$.

Lemma 2.1. Let $m \in \mathbb{N}(m \geq 2)$ and $u, x \in \mathbb{R}$ be fixed. Then we have

$$
h_{m, \nu}^{2}(1, u)=e^{i u} h_{m, \nu}^{2}(0, u) \quad(\nu=0,1)
$$

$$
\begin{aligned}
\frac{\partial^{j}}{\partial x^{j}} h_{m, \nu}^{2}(x \pm 1, u)= & e^{ \pm i u} \frac{\partial^{j}}{\partial x^{j}} h_{m, \nu}^{2}(x, u) \\
& (\nu=0,1 ; j=0, \ldots, m-2),
\end{aligned}
$$

$$
\begin{aligned}
& \frac{\partial}{\partial x} h_{m, 0}^{2}(x, u)=m\left(\frac{1}{\lfloor m / 2\rfloor} h_{m-1,0}^{2}(x, u)-\frac{1}{\lfloor(m+1) / 2\rfloor} h_{m-1,1}^{2}(x, u)\right), \\
& \frac{\partial}{\partial x} h_{m, 1}^{2}(x, u)=m\left(\frac{1}{\lfloor(m+1) / 2\rfloor} h_{m-1,1}^{2}(x, u)-\frac{e^{-i u}}{\lfloor m / 2\rfloor} h_{m-1,0}^{2}(x, u)\right) .
\end{aligned}
$$

For a proof we refer to [6].

Now for $x, x_{0}, x_{1}, u \in \mathbb{R}$ we define the following determinants:

with

$$
\begin{gathered}
h_{m}^{2}\left(x_{0}, x_{1}, u\right):=\operatorname{det}\left(\begin{array}{cc}
h_{m, 0}^{2}\left(x_{0}, u\right) & h_{m, 1}^{2}\left(x_{0}, u\right) \\
h_{m, 0}^{2}\left(x_{1}, u\right) & h_{m, 1}^{2}\left(x_{1}, u\right)
\end{array}\right), \\
h_{m}^{2}\left(x,\left[x_{0}, x_{1}\right], u\right):=\operatorname{det}\left(\begin{array}{cc}
h_{m, 0}^{2}(x, u) & h_{m, 1}^{2}(x, u) \\
h_{m, 0}^{2}\left[x_{0}, x_{1}\right](u) & h_{m, 1}^{2}\left[x_{0}, x_{1}\right](u)
\end{array}\right)
\end{gathered}
$$

$$
h_{m, \nu}^{2}\left[x_{0}, x_{1}\right](u):=\left[h_{m, \nu}^{2}(\cdot, u): x_{0}, x_{1}\right] \quad(\nu=0,1) .
$$

Note that the functions $h_{m, \nu}^{2}$ and $h_{m}^{2}$ are $2 \pi$-periodic in $u$.

Theorem 2.2. Let $N, m \in \mathbb{N}(m \geq 2)$ and $0<\tau_{0} \leq \tau_{1} \leq 1$ be given. Then the cardinal Hermite interpolation problem (1) is uniquely solvable if and only if

$$
h_{m}^{2}\left(\tau_{0},\left[\tau_{0}, \tau_{1}\right], u\right) \neq 0 \quad(u \in(-\pi, \pi]) .
$$

The relation (2) is equivalent to

$$
\left[B_{m}: \tau_{0}, \tau_{1}\right] \neq 0,
$$

where $B_{m}$ denotes the mth Bernoulli polynomial.

Proof. The spline function $s \in S_{m, 2}(\mathbb{Z})$ can be written in the form

$$
s(x)=\sum_{j=-\infty}^{\infty}\left(a_{j} N_{m, 0}^{2}(x-j)+b_{j} N_{m, 1}^{2}(x-j)\right) \quad(x \in \mathbb{R})
$$


with $\left\{a_{j}\right\}_{j=-\infty}^{\infty},\left\{b_{j}\right\}_{j=-\infty}^{\infty} \in l_{1}$. Then for $k \in \mathbb{Z}$, the interpolation conditions (1) read as follows:

$$
\begin{gathered}
\sum_{j=-\infty}^{\infty}\left(a_{j} N_{m, 0}^{2}\left(\tau_{0}+k-j\right)+b_{j} N_{m, 1}^{2}\left(\tau_{0}+k-j\right)\right)=y_{k}^{(0)}, \\
\sum_{j=-\infty}^{\infty}\left(a_{j}\left[N_{m, 0}^{2}(\cdot+k-j): \tau_{0}, \tau_{1}\right]+b_{j}\left[N_{m, 1}^{2}(\cdot+k-j): \tau_{0}, \tau_{1}\right]\right)=y_{k}^{(1)} .
\end{gathered}
$$

We introduce the following continuous functions defined on $(-\pi, \pi]$ :

$$
\begin{aligned}
\tilde{a}(u) & :=\sum_{j=-\infty}^{\infty} a_{j} e^{-i u j}, & \tilde{b}(u) & :=\sum_{j=-\infty}^{\infty} b_{j} e^{-i u j}, \\
\tilde{y}^{(0)}(u) & :=\sum_{j=-\infty}^{\infty} y_{j}^{(0)} e^{-i u j}, & \tilde{y}^{(1)}(u) & :=\sum_{j=-\infty}^{\infty} y_{j}^{(1)} e^{-i u j} .
\end{aligned}
$$

By (4) we find after a short calculation

$$
\left(\begin{array}{cc}
h_{m, 0}^{2}\left(\tau_{0}, u\right) & h_{m, 1}^{2}\left(\tau_{0}, u\right) \\
h_{m, 0}^{2}\left[\tau_{0}, \tau_{1}\right](u) & h_{m, 1}^{2}\left[\tau_{0}, \tau_{1}\right](u)
\end{array}\right)\left(\begin{array}{l}
\tilde{a} \\
\tilde{b}
\end{array}\right)=\left(\begin{array}{c}
\tilde{y}^{(0)}(u) \\
\tilde{y}^{(1)}(u)
\end{array}\right) .
$$

For given data $\mathbf{y}^{(0)}$ and $\mathbf{y}^{(1)}$, the functions $\tilde{a}$ and $\tilde{b}$ are uniquely determined by (1) if and only if

$$
h_{m}^{2}\left(\tau_{0},\left[\tau_{0}, \tau_{1}\right], u\right)=\operatorname{det}\left(\begin{array}{cc}
h_{m, 0}^{2}\left(\tau_{0}, u\right) & h_{m, 1}^{2}\left(\tau_{0}, u\right) \\
h_{m, 0}^{2}\left[\tau_{0}, \tau_{1}\right](u) & h_{m, 1}^{2}\left[\tau_{0}, \tau_{1}\right](u)
\end{array}\right)
$$

does not vanish on $(-\pi, \pi]$. The equivalence of $(2)$ and $(3)$ is shown in [5].

The function $h_{m}^{2}\left(\tau_{0},\left[\tau_{0}, \tau_{1}\right], \cdot\right)$ is called the symbol of the generalized Hermite spline interpolation problem (1).

Example 2.3. For $x, x_{0}, x_{1} \in(0,1], u \in \mathbb{R}$, we find in the case $m=2$, using

$$
h_{2,0}^{2}(x, u)=2 x(1-x), \quad h_{2,1}^{2}(x, u)=x^{2}+(1-x)^{2} e^{-i u},
$$

the symbol of the generalized Hermite spline interpolation problem

$$
h_{2}^{2}\left(x_{0},\left[x_{0}, x_{1}\right], u\right)=2\left(x_{0} x_{1}-\left(1-x_{0}\right)\left(1-x_{1}\right) e^{-i u}\right),
$$

and in the case $m=3$, using

$$
\begin{aligned}
& h_{3,0}^{2}(x, u)=\frac{1}{2}\left(x^{2}(6-5 x)+(1-x)^{3} e^{-i u}\right), \\
& h_{3,1}^{2}(x, u)=\frac{1}{2}\left(x^{3}+(1-x)^{2}(5 x+1) e^{-i u}\right),
\end{aligned}
$$

that

$$
\begin{aligned}
h_{3}^{2}\left(x_{0},\left[x_{0}, x_{1}\right], u\right) & \\
=\frac{3}{2}\left(x_{0}^{2} x_{1}^{2}-\left(x_{0}\left(1-x_{0}\right)+x_{1}\left(1-x_{1}\right)+\right.\right. & \left.2 x_{0} x_{1}\left(1-x_{0}\right)\left(1-x_{1}\right)\right) e^{-i u} \\
& \left.+\left(1-x_{0}\right)^{2}\left(1-x_{1}\right)^{2} e^{-2 i u}\right) .
\end{aligned}
$$

The following properties for the functions $h_{m}^{2}$ can be easily proved from the definition (see [6]). 
Lemma 2.4. Let $m \in \mathbb{N}(m \geq 2), x_{0}, x_{1} \in \mathbb{R}$, and $u \in(-\pi, \pi]$ be fixed. Then (i)

$$
\begin{aligned}
h_{m}^{2}\left(x_{0}, x_{1}, u\right) & =-h_{m}^{2}\left(x_{1}, x_{0}, u\right), \\
h_{m}^{2}\left(x_{0},\left[x_{0}, x_{1}\right], u\right) & =h_{m}^{2}\left(x_{1},\left[x_{0}, x_{1}\right], u\right),
\end{aligned}
$$

(ii)

$$
\begin{aligned}
h_{m}^{2}\left(x_{0}, x_{1}, u+2 k \pi\right) & =h_{m}^{2}\left(x_{0}, x_{1}, u\right) \quad(k \in \mathbb{Z}) \\
h_{m}^{2}\left(x_{0} \pm 1, x_{1}, u\right) & =e^{ \pm i u} h_{m}^{2}\left(x_{0}, x_{1}, u\right) \\
h_{m}^{2}\left(x_{0}, x_{1} \pm 1, u\right) & =e^{ \pm i u} h_{m}^{2}\left(x_{0}, x_{1}, u\right) \\
h_{m}^{2}\left(x_{0}, x_{0}, u\right) & =0
\end{aligned}
$$

(iii)

$$
\begin{aligned}
h_{m}^{2}\left(x_{0}+1,\left[x_{0}+1, x_{0}+1\right], u\right) & =e^{2 i u} h_{m}^{2}\left(x_{0},\left[x_{0}, x_{0}\right], u\right), \\
h_{m}^{2}\left(x_{0}-1,\left[x_{0}-1, x_{0}-1\right], u\right) & =e^{-2 i u} h_{m}^{2}\left(x_{0},\left[x_{0}, x_{0}\right], u\right), \\
h_{m}^{2}\left(x_{0},\left[x_{0}, x_{0}+k\right], u\right) & =0 \quad(k \in \mathbb{Z}) .
\end{aligned}
$$

For the computation of cardinal fundamental splines and cardinal Hermite spline interpolants by discrete Fourier transform we need the vectors

$$
\begin{array}{cc}
\mathbf{h}_{m, \nu}^{2}(x) & :=\left(h_{m, \nu}^{2}(x, 2 \pi j / N)\right)_{j=0}^{N-1} \quad(\nu=0,1 ; x \in \mathbb{R}), \\
\mathbf{h}_{m}^{2}\left(x_{0}, x_{1}\right):=\left(h_{m}^{2}\left(x_{0}, x_{1}, 2 \pi j / N\right)\right)_{j=0}^{N-1} & \left(x_{0}, x_{1} \in \mathbb{R}\right)
\end{array}
$$

and the corresponding divided differences with respect to $x$,

$$
\begin{aligned}
\mathbf{h}_{m, \nu}^{2}\left[x_{0}, x_{1}\right] & :=\left(h_{m, \nu}^{2}\left[x_{0}, x_{1}\right](2 \pi j / N)\right)_{j=0}^{N-1} \quad(\nu=0,1), \\
\mathbf{h}_{m}^{2}\left(x_{0},\left[x_{0}, x_{1}\right]\right) & :=\left(h_{m}^{2}\left(x_{0},\left[x_{0}, x_{1}\right], 2 \pi j / N\right)\right)_{j=0}^{N-1}
\end{aligned}
$$

with $x_{0}, x_{1} \in \mathbb{R}$.

We now present an efficient algorithm for the computation of $\mathbf{h}_{m, \nu}^{2}(x)(\nu=$ $0,1)$. Let the $N$-periodic B-splines $P_{m, \nu}^{2}$ of degree $m(m \geq 2)$ and defect 2 be defined by

$$
P_{m, \nu}^{2}(x):=\sum_{l=-\infty}^{\infty} N_{m, \nu}^{2}(x-l N) \quad(\nu=0,1 ; x \in \mathbb{R})
$$

We put

Then from

$$
\mathbf{n}_{m, \nu}(x):=\left(P_{m, \nu}^{2}(x+k)\right)_{k=0}^{N-1} \quad(\nu=0,1)
$$

$$
\sum_{k=0}^{N-1} P_{m, \nu}^{2}(x+k) w_{N}^{j k}=h_{m, \nu}^{2}(x, 2 \pi j / N) \quad(\nu=0,1 ; j=0, \ldots, N-1)
$$

with $w_{N}:=\exp (-2 \pi i / N)$ it follows that

$$
\mathbf{h}_{m, \nu}^{2}(x)=\mathbf{F}_{N} \mathbf{n}_{m, \nu}(x) \quad(\nu=0,1) .
$$

Here, $\mathbf{F}_{N}:=\left(w_{N}^{j k}\right)_{j, k=0}^{N-1}$ denotes the Fourier matrix of order $N$. We obtain: 
Algorithm 2.5. Computation of the auxiliary vectors $\mathbf{h}_{m, \nu}^{2}(x)(\nu=0,1)$

Input: $\quad m$ spline degree $(m \in \mathbb{N}, m \geq 2)$,

$N$ period $(N \in \mathbb{N}, N$ power of 2$)$,

$x \quad(x \in \mathbb{R})$.

1. Compute $P_{m, \nu}^{2}(x+j) \quad(\nu=0,1 ; j=0, \ldots, N-1)$ by the B-spline recursion formula. Put

$$
\mathbf{n}_{m, \nu}(x):=\left(P_{m, \nu}^{2}(x+j)\right)_{j=0}^{N-1} \in \mathbb{R}^{N}(\nu=0,1) \text {. }
$$

2. Compute $\mathbf{h}_{m, \nu}^{2}(x):=\mathbf{F}_{N} \mathbf{n}_{m, \nu}(x)(\nu=0,1)$ by fast Fourier transform. Output: $\mathbf{h}_{m, \nu}^{2}(x)=\left(h_{m, \nu}^{2}(x, 2 \pi j / N)\right)_{j=0}^{N-1}(\nu=0,1)$.

By the use of fast Fourier transform Algorithm 2.5 requires only $O(N \log N)$ arithmetic operations. The other vectors $\mathbf{h}_{m, \nu}^{2}\left[x_{0}, x_{1}\right](\nu=0,1), \mathbf{h}_{m}^{2}\left(x_{0}, x_{1}\right)$, and $\mathbf{h}_{m}^{2}\left(x_{0},\left[x_{0}, x_{1}\right]\right)$ can be computed in the same manner.

Remark 2.6. For solving the generalized Hermite spline interpolation problem for $r>2$, the Euler-Frobenius functions $h_{m, \nu}^{r}(\nu=0, \ldots, r-1)$ can be similarly introduced by using B-splines with $r$-fold knots.

\section{CARdinal fundamental SPLines}

With the help of B-splines with double knots we are able to find new explicit formulas for the cardinal fundamental splines (see Figures 1 and 2):

Theorem 3.1. Let $m \in \mathbb{N}(m \geq 2)$ and $0<\tau_{0} \leq \tau_{1} \leq 1$ be given such that $h_{m}^{2}\left(\tau_{0},\left[\tau_{0}, \tau_{1}\right], u\right) \neq 0 \quad(u \in(-\pi, \pi])$ is satisfied. Then $L_{m, \nu} \in S_{m, 2}(\mathbb{Z})$, where

$$
\begin{array}{ll}
L_{m, 0}(x):=\frac{1}{2 \pi} \int_{-\infty}^{\infty} \frac{D_{m, 0}\left(\tau_{0}, \tau_{1}, u\right)}{h_{m}^{2}\left(\tau_{0},\left[\tau_{0}, \tau_{1}\right], u\right)} e^{i u x} d u & (x \in \mathbb{R}), \\
L_{m, 1}(x):=\frac{1}{2 \pi} \int_{-\infty}^{\infty} \frac{D_{m, 1}\left(\tau_{0}, \tau_{1}, u\right)}{h_{m}^{2}\left(\tau_{0},\left[\tau_{0}, \tau_{1}\right], u\right)} e^{i u x} d u & (x \in \mathbb{R})
\end{array}
$$

with

$$
\begin{aligned}
& D_{m, 0}\left(\tau_{0}, \tau_{1}, u\right):=\operatorname{det}\left(\begin{array}{cc}
\left(N_{m, 0}^{2}\right)^{\wedge}(u) & \left(N_{m, 1}^{2}\right)^{\wedge}(u) \\
h_{m, 0}^{2}\left[\tau_{0}, \tau_{1}\right](u) & h_{m, 1}^{2}\left[\tau_{0}, \tau_{1}\right](u)
\end{array}\right) \quad(u \in \mathbb{R}), \\
& D_{m, 1}\left(\tau_{0}, \tau_{1}, u\right):=\operatorname{det}\left(\begin{array}{cc}
h_{m, 0}^{2}\left(\tau_{0}, u\right) & h_{m, 1}^{2}\left(\tau_{0}, u\right) \\
\left(N_{m, 0}^{2}\right)^{\wedge}(u) & \left(N_{m, 1}^{2}\right)^{\wedge}(u)
\end{array}\right) \quad(u \in \mathbb{R}),
\end{aligned}
$$

are the cardinal fundamental splines of the Hermite spline interpolation problem (1), i.e., there holds

$$
\begin{aligned}
& L_{m, 0}\left(k+\tau_{0}\right)=\delta_{0, k} \quad(k \in \mathbb{Z}), \quad\left[L_{m, 0}(k+\cdot): \tau_{0}, \tau_{1}\right]=0 \quad(k \in \mathbb{Z}), \\
& L_{m, 1}\left(k+\tau_{0}\right)=0 \quad(k \in \mathbb{Z}), \quad\left[L_{m, 1}(k+\cdot): \tau_{0}, \tau_{1}\right]=\delta_{0, k} \quad(k \in \mathbb{Z}) .
\end{aligned}
$$

The coefficients $l_{j, \nu, 0}, l_{j, \nu, 1}(\nu=0,1)$ in the representation of $L_{m, \nu}$ by

$$
L_{m, \nu}(x)=\sum_{j=-\infty}^{\infty}\left(l_{j, \nu, 0} N_{m, 0}^{2}(x-j)+l_{j, \nu, 1} N_{m, 1}^{2}(x-j)\right) \quad(\nu=0,1 ; x \in \mathbb{R})
$$


are given by

$$
\begin{aligned}
& l_{j, 0,0}=\frac{1}{2 \pi} \int_{-\pi}^{\pi} \frac{h_{m, 1}^{2}\left[\tau_{0}, \tau_{1}\right](u)}{h_{m}^{2}\left(\tau_{0},\left[\tau_{0}, \tau_{1}\right], u\right)} e^{i u j} d u, \\
& l_{j, 0,1}=-\frac{1}{2 \pi} \int_{-\pi}^{\pi} \frac{h_{m, 0}^{2}\left[\tau_{0}, \tau_{1}\right](u)}{h_{m}^{2}\left(\tau_{0},\left[\tau_{0}, \tau_{1}\right], u\right)} e^{i u j} d u, \\
& l_{j, 1,0}=-\frac{1}{2 \pi} \int_{-\pi}^{\pi} \frac{h_{m, 1}^{2}\left(t_{0}, u\right)}{h_{m}^{2}\left(\tau_{0},\left[\tau_{0}, \tau_{1}\right], u\right)} e^{i u j} d u, \\
& l_{j, 1,1}=\frac{1}{2 \pi} \int_{-\pi}^{\pi} \frac{h_{m, 0}^{2}\left(\tau_{0}, u\right)}{h_{m}^{2}\left(\tau_{0},\left[\tau_{0}, \tau_{1}\right], u\right)} e^{i u j} d u .
\end{aligned}
$$

Proof. Since the trigonometric polynomial $h_{m}^{2}\left(\tau_{0},\left[\tau_{0}, \tau_{1}\right], \cdot\right)$ satisfies the condition $h_{m}^{2}\left(\tau_{0},\left[\tau_{0}, \tau_{1}\right], u\right) \neq 0(u \in(-\pi, \pi])$, there exists $\alpha>0$ such that $\left|h_{m}^{2}\left(\tau_{0},\left[\tau_{0}, \tau_{1}\right], u\right)\right| \geq \alpha>0$ for all $u \in \mathbb{R}$. Further, $N_{m, \nu}^{2} \in C^{m-2}(\mathbb{R}) \quad(\nu=$ $0,1)$ yields $\left(N_{m, \nu}^{2}\right)^{\wedge}(u)=O\left(|u|^{-m+1}\right) \quad(|u| \rightarrow \infty)$, i.e., $\left(N_{m, \nu}^{2}\right)^{\wedge} \quad(\nu=0,1)$ are absolutely integrable for $m \geq 3$. For $m=2$ this assertion follows from Example 4.2. Hence, the functions $L_{m, 0}$ and $L_{m, 1}$ are well defined and continuous.

1. We show that $\left(L_{m, 0}\left(\cdot+\tau_{0}\right)\right)^{\sim} \equiv 1$ and $l^{\sim} \equiv 0$, where $l(x):=$ $\left[L_{m, 0}(x+\cdot): \tau_{0}, \tau_{1}\right]$. By $(5)$ we find

$$
L_{m, 0}^{\wedge}(u)=\frac{D_{m, 0}\left(\tau_{0}, \tau_{1}, u\right)}{h_{m}^{2}\left(\tau_{0},\left[\tau_{0}, \tau_{1}\right], u\right)}
$$

Using the Poisson summation formula, we have

$$
\begin{aligned}
\left(N_{m, \nu}^{2}(\cdot+r)\right)^{\sim}(u)=\sum_{k=-\infty}^{\infty} N_{m, \nu}^{2}(k+\tau) e^{i u k}=h_{m, \nu}^{2}(\tau, u) & \\
& (\nu=0,1 ; \tau, u \in \mathbb{R}) .
\end{aligned}
$$

Consequently,

$$
\begin{gathered}
\left(L_{m, 0}\left(\cdot+\tau_{0}\right)\right)^{\sim}(u)=\sum_{j=-\infty}^{\infty}\left(\frac{\left(N_{m, 0}^{2}\left(\cdot+\tau_{0}\right)\right)^{\wedge}(u+2 \pi j) h_{m, 1}^{2}\left[\tau_{0}, \tau_{1}\right](u)}{h_{m}^{2}\left(\tau_{0},\left[\tau_{0}, \tau_{1}\right], u\right)}\right. \\
\left.-\frac{\left(N_{m, 1}^{2}\left(\cdot+\tau_{0}\right)\right)^{\wedge}(u+2 \pi j) h_{m, 0}^{2}\left[\tau_{0}, \tau_{1}\right](u)}{h_{m}^{2}\left(\tau_{0},\left[\tau_{0}, \tau_{1}\right], u\right)}\right) \\
=\frac{h_{m, 0}^{2}\left(\tau_{0}, u\right) h_{m, 1}^{2}\left[\tau_{0}, \tau_{1}\right](u)-h_{m, 1}^{2}\left(\tau_{0}, u\right) h_{m, 0}^{2}\left[\tau_{0}, \tau_{1}\right](u)}{h_{m}^{2}\left(\tau_{0},\left[\tau_{0}, \tau_{1}\right], u\right)}=1
\end{gathered}
$$

for all $u \in \mathbb{R}$. With

$$
n_{m, \nu}^{2}(x):=\left[N_{m, \nu}^{2}(x+\cdot): \tau_{0}, \tau_{1}\right] \quad(\nu=0,1 ; x \in \mathbb{R})
$$

we obtain for $u \in \mathbb{R}$ that 


$$
\begin{aligned}
l^{\sim}(u) & =\sum_{j=-\infty}^{\infty}\left(\frac{\left(n_{m, 0}^{2}\right)^{\wedge}(u+2 \pi j) h_{m, 1}^{2}\left[\tau_{0}, \tau_{1}\right](u)}{h_{m}^{2}\left(\tau_{0},\left[\tau_{0}, \tau_{1}\right], u\right)}\right. \\
\left.-\frac{\left(n_{m, 1}^{2}\right)^{\wedge}(u+2 \pi j) h_{m, 0}^{2}\left[\tau_{0}, \tau_{1}\right](u)}{h_{m}^{2}\left(\tau_{0},\left[\tau_{0}, \tau_{1}\right], u\right)}\right) & \\
= & \frac{h_{m, 0}^{2}\left[\tau_{0}, \tau_{1}\right](u) h_{m, 1}^{2}\left[\tau_{0}, \tau_{1}\right](u)-h_{m, 1}^{2}\left[\tau_{0}, \tau_{1}\right](u) h_{m, 0}^{2}\left[\tau_{0}, \tau_{1}\right](u)}{h_{m}^{2}\left(\tau_{0},\left[\tau_{0}, \tau_{1}\right], u\right)}=0 .
\end{aligned}
$$

Thus, we have for all $k \in \mathbb{Z}$

$$
\begin{aligned}
L_{m, 0}\left(k+\tau_{0}\right) & =\frac{1}{2 \pi} \int_{-\infty}^{\infty}\left(L_{m, 0}\left(\cdot+\tau_{0}\right)\right)^{\wedge}(u) e^{i u k} d u \\
& =\frac{1}{2 \pi} \int_{-\pi}^{\pi}\left(L_{m, 0}\left(\cdot+\tau_{0}\right)\right)^{\sim}(u) e^{i u k} d u=\delta_{0, k}
\end{aligned}
$$

and

$$
\left[L_{m, 0}(k+\cdot): \tau_{0}, \tau_{1}\right]=\frac{1}{2 \pi} \int_{-\infty}^{\infty} l^{\wedge}(u) e^{i u k} d u=\frac{1}{2 \pi} \int_{-\pi}^{\pi} l^{\sim}(u) e^{i u k} d u=0 .
$$

2. Using the Poisson summation formula, we obtain

$$
\begin{aligned}
& L_{m, 0}(x)= \frac{1}{2 \pi} \int_{-\infty}^{\infty} \frac{D_{m, 0}\left(\tau_{0}, \tau_{1}, u\right)}{h_{m}^{2}\left(\tau_{0},\left[\tau_{0}, \tau_{1}\right], u\right)} e^{i u x} d u \\
&= \frac{1}{2 \pi} \int_{-\pi}^{\pi} \frac{1}{h_{m}^{2}\left(\tau_{0},\left[\tau_{0}, \tau_{1}\right], u\right)} \\
& \times\left(\sum_{j=-\infty}^{\infty}\left(N_{m, 0}^{2}\right)^{\wedge}(u+2 \pi j) h_{m, 1}^{2}\left[\tau_{0}, \tau_{1}\right](u) e^{i(u+2 \pi j) x}\right. \\
&\left.-\sum_{j=-\infty}^{\infty}\left(N_{m, 1}^{2}\right)^{\wedge}(u+2 \pi j) h_{m, 0}^{2}\left[\tau_{0}, \tau_{1}\right](u) e^{i(u+2 \pi j) x}\right) d u \\
&= \frac{1}{2 \pi} \int_{-\pi}^{\pi}\left(\frac{\left(\sum_{k=-\infty}^{\infty} N_{m, 0}^{2}(k+x) e^{-i u k}\right) h_{m, 1}^{2}\left[\tau_{0}, \tau_{1}\right](u)}{h_{m}^{2}\left(\tau_{0},\left[\tau_{0}, \tau_{1}\right], u\right)}\right. \\
&\left.\quad-\frac{\left(\Sigma_{k=-\infty}^{\infty} N_{m, 1}^{2}(k+x) e^{-i u k}\right) h_{m, 0}^{2}\left[\tau_{0}, \tau_{1}\right](u)}{h_{m}^{2}\left(\tau_{0},\left[\tau_{0}, \tau_{1}\right], u\right)} d u\right) \\
&=\sum_{j=-\infty}^{\infty}\left(l_{j, 0,0} N_{m, 0}^{2}(x-j)+l_{j, 0,1} N_{m, 1}^{2}(x-j)\right)
\end{aligned}
$$

with

$$
\begin{aligned}
& l_{j, 0,0}=\frac{1}{2 \pi} \int_{-\pi}^{\pi} \frac{h_{m, 1}^{2}\left[\tau_{0}, \tau_{1}\right](u)}{h_{m}^{2}\left(\tau_{0},\left[\tau_{0}, \tau_{1}\right], u\right)} e^{i u j} d u, \\
& l_{j, 0,1}=-\frac{1}{2 \pi} \int_{-\pi}^{\pi} \frac{h_{m, 0}^{2}\left[\tau_{0}, \tau_{1}\right](u)}{h_{m}^{2}\left(\tau_{0},\left[\tau_{0}, \tau_{1}\right], u\right)} e^{i u j} d u .
\end{aligned}
$$

The assertion for $L_{m, 1}$ can be proved analogously. 


\section{SOLUTION OF THE CARDINAL HERMITE SPLINE INTERPOLATION PROBLEM}

Let $m \in \mathbb{N}(m \geq 2)$ and $0<\tau_{0} \leq \tau_{1} \leq 1$ be given such that

$$
h_{m}^{2}\left(\tau_{0},\left[\tau_{0}, \tau_{1}\right], u\right) \neq 0 \quad(u \in(-\pi, \pi]) .
$$

If $\mathbf{y}^{(0)}:=\left\{y_{j}^{(0)}\right\}_{j=-\infty}^{\infty}, \mathbf{y}^{(1)}:=\left\{y_{j}^{(1)}\right\}_{j=-\infty}^{\infty} \in l_{1}$ are given data sequences, then a continuous solution $s \in S_{m, 2}(\mathbb{Z}) \quad(m \geq 2)$ of the cardinal Hermite spline interpolation problem (1) can be written as follows:

$$
s(x)=\sum_{j=-\infty}^{\infty}\left(y_{j}^{(0)} L_{m, 0}(x-j)+y_{j}^{(1)} L_{m, 1}(x-j)\right) .
$$

This series is absolutely and uniformly convergent on $\mathbb{R}$. Using the properties of Fourier transform, we find

$$
s^{\wedge}(u)=L_{m, 0}^{\wedge}(u) \sum_{l=-\infty}^{\infty} y_{l}^{(0)} e^{-i u l}+L_{m, 1}^{\wedge}(u) \sum_{l=-\infty}^{\infty} y_{l}^{(1)} e^{-i u l} \quad(u \in \mathbb{R}) .
$$

For the $r$ th derivative $s^{(r)}(1 \leq r \leq m-2)$ there follows

$$
\begin{aligned}
\left(s^{(r)}\right)^{\wedge}(u) & =(i u)^{r} s^{\wedge}(u) \\
& =(i u)^{r}\left(L_{m, 0}^{\wedge}(u) \sum_{l=-\infty}^{\infty} y_{l}^{(0)} e^{-i u l}+L_{m, 1}^{\wedge}(u) \sum_{l=-\infty}^{\infty} \cdot y_{l}^{(1)} e^{-i u l}\right)
\end{aligned}
$$

with $u \in \mathbb{R}$. Since $(i \cdot)^{m-2} L_{m, \nu}^{\wedge} \in L_{1}(\mathbb{R}) \quad(\nu=0,1)$, we find that $\left(s^{(r)}\right)^{\wedge} \in$ $L_{1}(\mathbb{R})$. Inverse Fourier transform yields

$$
s^{(r)}(x)=\frac{1}{2 \pi} \int_{-\infty}^{\infty}(i u)^{r} s^{\wedge}(u) e^{i u x} d u .
$$

We shall compute this solution $s$ and its derivatives $s^{(r)}(1 \leq r \leq m-2)$ by means of fast Fourier transform.

Assume that $y_{l}^{(0)}=0, y_{l}^{(1)}=0$ for $l \notin\{0, \ldots, N-1\}$. This assumption makes sense, since $\mathbf{y}^{(\nu)} \in l_{1} \quad(\nu=0,1)$. Replacing in (7) the integration over $\mathbb{R}$ by integration over $[-\mu \pi, \mu \pi](\mu \in \mathbb{N})$, i.e.,

$$
\frac{1}{2 \pi} \int_{-\mu \pi}^{\mu \pi}\left(L_{m, 0}^{\wedge}(u) \sum_{l=0}^{N-1} y_{l}^{(0)} e^{-i l u}+L_{m, 1}^{\wedge}(u) \sum_{l=0}^{N-1} y_{l}^{(1)} e^{-i l u}\right)(i u)^{r} e^{i u x} d u
$$

we compute (8) by the rectangle rule with step length $2 \pi / N$ :

$$
\begin{aligned}
\frac{1}{N} \sum_{n \in G_{\mu N}}\left(L_{m, 0}^{\wedge}\left(\frac{2 \pi n}{N}\right) \sum_{l=0}^{N-1} y_{l}^{(0)} w_{N}^{l n}\right. \\
\left.+L_{m, 1}^{\wedge}\left(\frac{2 \pi n}{N}\right) \sum_{l=0}^{N-1} y_{l}^{(1)} w_{N}^{l n}\right)\left(\frac{2 \pi i n}{N}\right)^{r} e^{2 \pi i x n / N}
\end{aligned}
$$


with $G_{\mu N}:=\{n \in \mathbb{Z}: \mu N / 2<n \leq \mu N / 2\}$ and $w_{N}:=\exp (-2 \pi i / N)$. Instead of $s^{(r)}(j / \mu) \quad(j=0, \ldots, \mu N-1)$ we obtain the approximate value

$$
\begin{aligned}
s_{j}^{r}:=\frac{1}{N} \sum_{n \in G_{\mu N}}\left(\frac{2 \pi i n}{N}\right)^{r}\left(L_{m, 0}^{\wedge}\left(\frac{2 \pi n}{N}\right) \sum_{l=0}^{N-1} y_{l}^{(0)} w_{N}^{l n}\right. \\
\left.+L_{m, 1}^{\wedge}\left(\frac{2 \pi n}{N}\right) \sum_{l=0}^{N-1} y_{l}^{(1)} w_{N}^{l n}\right) w_{\mu N}^{-n j} .
\end{aligned}
$$

Note from (5) that

$$
L_{m, \nu}^{\wedge}(u)=\frac{D_{m, \nu}\left(\tau_{0}, \tau_{1}, u\right)}{h_{m}^{2}\left(\tau_{0},\left[\tau_{0}, \tau_{1}\right], u\right)} \quad(\nu=0,1)
$$

with $D_{m, \nu}$ as in Theorem 3.1.

Thus, we find the following algorithm for the computation of the cardinal Hermite spline interpolant $s \in S_{m, 2}(\mathbb{Z})$ and its derivatives:

Algorithm 4.1. Computation of the cardinal Hermite spline interpolant and its derivatives

Input : $m$

$N$

$\mu$

$\mathbf{y}^{(0)}, \mathbf{y}^{(1)}$

$r$

$\tau_{0}, \tau_{1}$ spline degree $(m \in \mathbb{N}, m \geq 2)$,

power of 2 ,

power of 2 ,

data sequences with $y_{j}^{(\nu)}=0(j \notin\{0, \ldots, N-1\}$;

$$
\nu=0,1) \text {, }
$$

order of a derivative $(0 \leq r \leq m-2)$,

shift parameters $\left(0<\tau_{0} \leq \tau_{1} \leq 1\right)$ with

$\left[B_{m}: \tau_{0}, \tau_{1}\right] \neq 0$,

$\left(N_{m, 0}^{2}\right)^{\wedge},\left(N_{m, 1}^{2}\right)^{\wedge}$ Fourier transforms of $N_{m, 0}^{2}$ and $N_{m, 1}^{2}$.

1. Precompute for $\nu=0,1$ the vectors

$$
\begin{gathered}
\left(h_{m, \nu}^{2}\left(\tau_{0}, 2 \pi l / N\right)\right)_{l=0}^{N-1}, \quad\left(h_{m, \nu}^{2}\left[\tau_{0}, \tau_{1}\right](2 \pi l / N)\right)_{l=0}^{N-1}, \\
\left(h_{m}^{2}\left(\tau_{0},\left[\tau_{0}, \tau_{1}\right], 2 \pi l / N\right)\right)_{l=0}^{N-1}
\end{gathered}
$$

by Algorithm 2.5 and by the definitions.

2. Precompute by (6) for all $n \in G_{\mu N}$

$$
D_{m, 0}\left(\tau_{0}, \tau_{1}, 2 \pi n / N\right), \quad D_{m, 1}\left(\tau_{0}, \tau_{1}, 2 \pi n / N\right) .
$$

3. Compute by fast Fourier transform

$$
\hat{y}_{j}^{(0)}:=\sum_{k=0}^{N-1} y_{k}^{(0)} w_{N}^{j k}, \quad \hat{y}_{j}^{(1)}:=\sum_{k=0}^{N-1} y_{k}^{(1)} w_{N}^{j k} \quad(j=0, \ldots, N-1) .
$$

4. Form for all $n \in G_{\mu N}$

$$
t_{n}^{r}:=\left(\frac{2 \pi i n}{N}\right)^{r} \frac{D_{m, 0}\left(\tau_{0}, \tau_{1}, 2 \pi n / N\right) \hat{y}_{n^{\prime}}^{(0)}+D_{m, 1}\left(\tau_{0}, \tau_{1}, 2 \pi n / N\right) \hat{y}_{n^{\prime}}^{(1)}}{h_{m}^{2}\left(\tau_{0},\left[\tau_{0}, \tau_{1}\right], 2 \pi n^{\prime} / N\right)}
$$




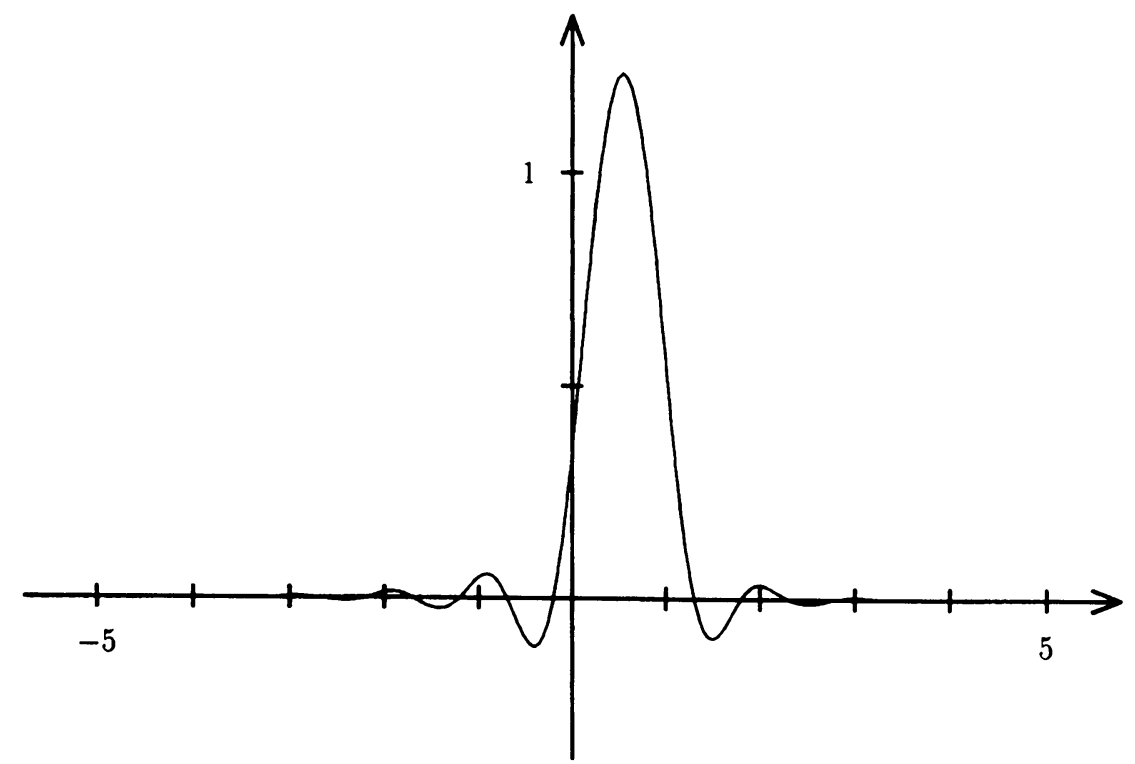

FIGURE 1. Cardinal fundamental spline $L_{5,0}$ for the shift parameters $\tau_{0}=0.3, \tau_{1}=0.8$

with $n^{\prime}:=n \bmod N$, i.e., $n^{\prime}$ is the nonnegative residue of $n$ modulo $N$ defined by $n^{\prime} \equiv n(\bmod N)$ and $0 \leq n^{\prime} \leq N-1$.

5. Put for all $n \in G_{\mu N}$ with $k:=n \bmod (\mu N)$

$$
v_{k}^{r}:=t_{n}^{r}
$$

6. Compute by fast Fourier transform

$$
s_{j}^{r}:=\frac{1}{N} \sum_{k=0}^{\mu N-1} v_{k}^{r} w_{\mu N}^{-j k} \quad(j=0, \ldots, \mu N-1) .
$$

Output: $s_{j}^{r}$ approximate value of $s^{(r)}(j / \mu)(j=0, \ldots, \mu N-1)$. The cardinal fundamental splines $L_{5,0}$ and $L_{5,1}$ for $\tau_{0}=0.3, \tau_{1}=0.8$ (see Figures 1 and 2) are obtained by Algorithm 4.1 .

The main operations of this algorithm are two discrete Fourier transforms of length $N$ in step 3, and the discrete Fourier transform of length $\mu N$ in step 6. Since we use fast Fourier transform, the algorithm possesses an arithmetical complexity of $O(\mu N(\log \mu N))$ and computes $\mu N$ approximate values of $s^{(r)}$ all at once. The numerical stability of the algorithm is mainly determined by

$$
\min \left\{\left|h_{m}^{2}\left(\tau_{0},\left[\tau_{0}, \tau_{1}\right], 2 \pi k / N\right)\right| ; k=0, \ldots, N-1\right\},
$$

because in step 4 one has to divide by this quantity.

The Fourier transforms of $N_{m, \nu}^{2}(\nu=0,1)$ can be easily precomputed, since the B-splines have a compact support. 


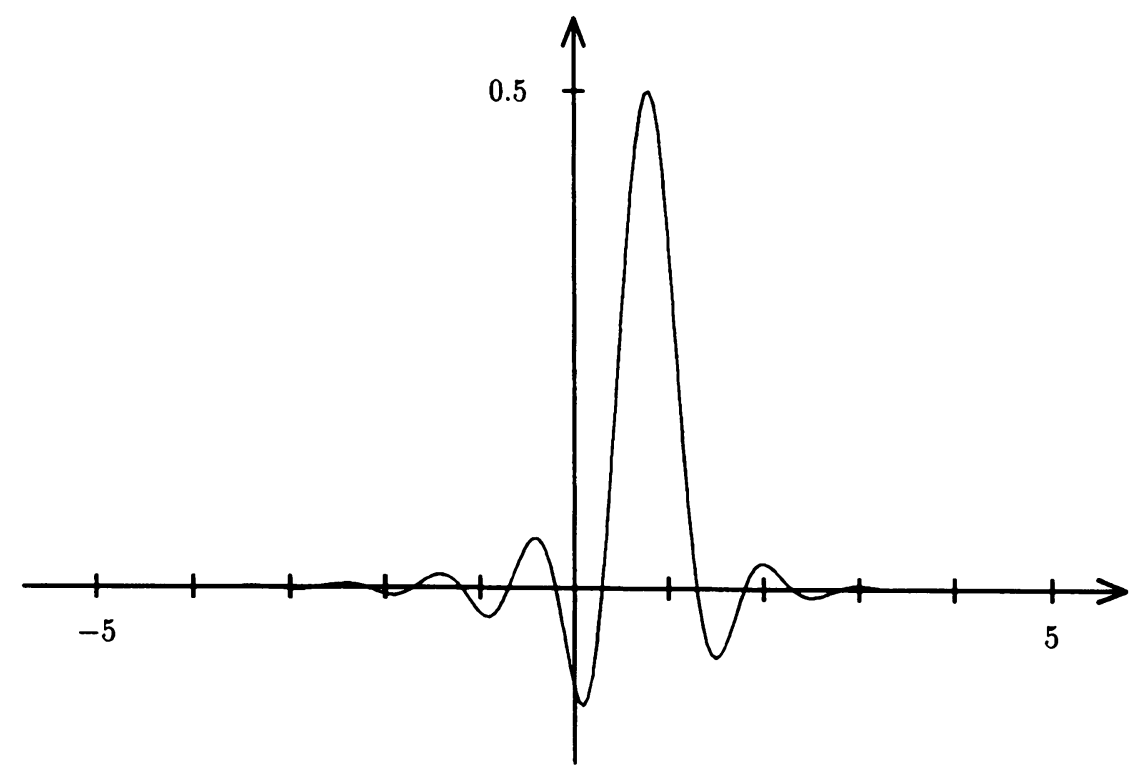

Figure 2. Cardinal fundamental spline $L_{5,1}$ for the shift parameters $\tau_{0}=0.3, \tau_{1}=0.8$

Example 4.2. The Fourier transforms of $N_{m, \nu}^{2}(m=2,3 ; \nu=0,1)$, for $u \neq 0$, are

$$
\begin{aligned}
& \left(N_{2,0}^{2}\right)^{\wedge}(u)=\frac{2}{u^{3}}\left((-u+2 i) e^{-i u}-u-2 i\right), \\
& \left(N_{2,1}^{2}\right)^{\wedge}(u)=\frac{2}{u^{3}}\left(-i e^{-2 i u}+2 u e^{-i u}+i\right), \\
& \left(N_{3,0}^{2}\right)^{\wedge}(u)=\frac{3}{u^{4}}\left(e^{-2 i u}+4(i u+1) e^{-i u}+2 i u-5\right), \\
& \left(N_{3,1}^{2}\right)^{\wedge}(u)=\frac{3}{u^{4}}\left((-2 i u-5) e^{-2 i u}+(-i u+1) e^{-i u}+1\right) .
\end{aligned}
$$

\section{Periodic Hermite spline interpolation}

Finally, we want to show a close connection between periodic and cardinal spline interpolation. We shall find that Algorithm 4.1 can also be used for the computation of the periodic Hermite spline interpolant and its derivatives.

Let $N, m \in \mathbb{N}(m \geq 2)$ be fixed. By $S_{m, 2}^{N}(\mathbb{Z})$ we denote the set of all functions

$$
s(x):=\sum_{j=0}^{N-1}\left(a_{j} P_{m, 0}^{2}(x-j)+b_{j} P_{m, 1}^{2}(x-j)\right) \quad(x \in \mathbb{R})
$$

with arbitrary coefficients $a_{j}, b_{j} \in \mathbb{R}(j=0, \ldots, N-1)$. Furthermore, let $y_{j}^{(\nu)} \in \mathbb{R} \quad(\nu=0,1 ; j \in \mathbb{Z})$ with $y_{j}^{(\nu)}=y_{j+N}^{(\nu)}$ be given $N$-periodic data, which can be completely described by the vectors

$$
\mathbf{y}^{(\nu)}:=\left(y_{0}^{(\nu)}, \ldots, y_{N-1}^{(\nu)}\right)^{T} \in \mathbb{R}^{N} \quad(\nu=0,1) .
$$

We consider the following generalized $N$-periodic Hermite spline interpolation problem: For given shift parameters $\tau_{0}, \tau_{1} \in \mathbb{R}$ with $0<\tau_{0} \leq \tau_{1} \leq 1$, find an 
$N$-periodic spline function $s \in S_{m, 2}^{N}(\mathbb{Z})$ such that

$$
s\left(j+\tau_{0}\right)=y_{j}^{(0)}, \quad\left[s(j+\cdot): \tau_{0}, \tau_{1}\right]=y_{j}^{(1)} \quad(j \in \mathbb{Z}) .
$$

In [5] it is shown that the periodic Hermite spline interpolation problem is uniquely solvable if and only if

$$
h_{m}^{2}\left(\tau_{0},\left[\tau_{0}, \tau_{1}\right], 2 \pi n / N\right) \neq 0 \quad(n=0, \ldots, N-1) .
$$

Again, this condition is equivalent to (3). Let $L_{m, \nu}^{N} \in S_{m, 2}^{N}(\mathbb{Z}) \quad(\nu=0,1)$ be the $N$-periodic fundamental splines for the interpolation problem (10), i.e., there holds

$$
\begin{aligned}
& L_{m, 0}^{N}\left(k+\tau_{0}\right)=\delta_{0, k}^{N}, \quad\left[L_{m, 0}^{N}(k+\cdot): \tau_{0}, \tau_{1}\right]=0 \quad(k \in \mathbb{Z}), \\
& L_{m, 1}^{N}\left(k+\tau_{0}\right)=0, \quad\left[L_{m, 1}^{N}(k+\cdot): \tau_{0}, \tau_{1}\right]=\delta_{0, k}^{N} \quad(k \in \mathbb{Z})
\end{aligned}
$$

with

$$
\delta_{0, k}^{N}:= \begin{cases}1, & k \equiv 0(\bmod N), \\ 0, & k \neq 0(\bmod N) .\end{cases}
$$

The $N$-periodic fundamental splines $L_{m, \nu}^{N}$ can be represented as follows (cf. [6]):

$$
\begin{array}{ll}
L_{m, 0}^{N}(x):=\frac{1}{N} \sum_{j=0}^{N-1} \frac{h_{m}^{2}\left(x,\left[\tau_{0}, \tau_{1}\right], 2 \pi j / N\right)}{h_{m}^{2}\left(\tau_{0},\left[\tau_{0}, \tau_{1}\right], 2 \pi j / N\right)} & (x \in \mathbb{R}), \\
L_{m, 1}^{N}(x):=\frac{1}{N} \sum_{j=0}^{N-1} \frac{h_{m}^{2}\left(\tau_{0}, x, 2 \pi j / N\right)}{h_{m}^{2}\left(\tau_{0},\left[\tau_{0}, \tau_{1}\right], 2 \pi j / N\right)} \quad(x \in \mathbb{R}) .
\end{array}
$$

Assume that (3) is satisfied. Then the following connection between $N$ periodic and cardinal fundamental splines can be observed:

$$
L_{m, \nu}^{N}(x)=\sum_{l=-\infty}^{\infty} L_{m, \nu}(x+l N) \quad(\nu=0,1) .
$$

Since $L_{m, \nu} \in L_{1}(\mathbb{R})$, the series is absolutely and uniformly convergent on $\mathbb{R}$. For the Fourier coefficients of $L_{m, \nu}^{N}$ with respect to the orthogonal system $\left\{e^{2 \pi i k \cdot / N} ; k \in \mathbb{Z}\right\}$ we get

$$
\begin{aligned}
c_{k}\left(L_{m, \nu}^{N}\right) & :=\frac{1}{N} \int_{0}^{N} L_{m, \nu}^{N}(x) e^{-2 \pi i k x / N} d x \\
& =\frac{1}{N} \int_{0}^{N} \sum_{l=-\infty}^{\infty} L_{m, \nu}(x+l N) e^{-2 \pi i k x / N} d x \\
& =\frac{1}{N} \int_{-\infty}^{\infty} L_{m, \nu}(x) e^{-2 \pi i k x / N} d x=\frac{1}{N} L_{m, \nu}^{\wedge}(2 \pi k / N) .
\end{aligned}
$$

Now for the computation of the $N$-periodic fundamental splines and their derivatives we can start from

$$
\begin{aligned}
\left(L_{m, \nu}^{N}\right)^{(r)}(x) & =\sum_{l=-\infty}^{\infty}(2 \pi i l / N)^{r} c_{l}\left(L_{m, \nu}^{N}\right) e^{2 \pi i l x / N} \\
& =\frac{1}{N} \sum_{l=-\infty}^{\infty}(2 \pi i l / N)^{r} L_{m, \nu}^{\wedge}(2 \pi l / N) e^{2 \pi i l x / N}
\end{aligned}
$$


We consider the truncated sum

$$
\frac{1}{N} \sum_{n \in G_{\mu N}}(2 \pi i n / N)^{r} L_{m, \nu}^{\wedge}(2 \pi n / N) e^{2 \pi i x n / N}
$$

and obtain the following approximate value of $\left(L_{m, \nu}^{N}\right)^{(r)}(k / \mu) \quad\left(k \in G_{\mu N}\right)$ :

$$
\left(l_{\nu, k}^{N}\right)^{r}:=\frac{1}{N} \sum_{n \in G_{\mu N}}(2 \pi i n / N)^{r} L_{m, \nu}^{\wedge}(2 \pi n / N) e^{2 \pi i k n /(\mu N)} .
$$

With the help of the $N$-periodic fundamental splines $L_{m, \nu}^{N}(\nu=0,1)$, a continuous solution $s \in S_{m, 2}^{N}(\mathbb{Z})$ of the $N$-periodic Hermite spline interpolation problem (10) can be written as follows:

$$
s(x)=\sum_{j=0}^{N-1}\left(y_{j}^{(0)} L_{m, 0}^{N}(x-j)+y_{j}^{(1)} L_{m, 1}^{N}(x-j)\right),
$$

where $\mathbf{y}^{(\nu)}:=\left(y_{0}^{(\nu)}, \ldots, y_{N-1}^{(\nu)}\right)^{T} \in \mathbb{R}^{N}(\nu=0,1)$ are given data vectors. By

$$
\left(L_{m, \nu}^{N}(x-j)\right)^{(r)} \approx \frac{1}{N} \sum_{n \in G_{\mu N}}\left(\frac{2 \pi i n}{N}\right)^{r} L_{m, \nu}^{\wedge}(2 \pi n / N) e^{2 \pi i n x / N} w_{N}^{n j}
$$

we find

$$
\begin{aligned}
s^{(r)}(x) \approx \frac{1}{N} \sum_{n \in G_{\mu N}}\left(\frac{2 \pi i n}{N}\right)^{r}\left(L_{m, 0}^{\wedge}(2 \pi n / N) \sum_{l=0}^{N-1} y_{l}^{(0)} w_{N}^{l n^{\prime}}\right. \\
\left.+L_{m, 1}^{\wedge}(2 \pi n / N) \sum_{l=0}^{N-1} y_{l}^{(1)} w_{N}^{l n^{\prime}}\right) e^{2 \pi i x n / N}
\end{aligned}
$$

with $n^{\prime}:=n \bmod N$. That means, instead of $s^{(r)}(j / \mu) \quad(j=0,1, \ldots, \mu N-$ $1 ; r=0, \ldots, m-1)$ we obtain the approximate value

$$
\begin{aligned}
s_{j}^{r}:=\frac{1}{N} \sum_{n \in G_{\mu N}}\left(\frac{2 \pi i n}{N}\right)^{r}\left(L_{m, 0}^{\wedge}(2 \pi n / N)\right. & \sum_{k=0}^{N-1} y_{k}^{(0)} w_{N}^{k n^{\prime}} \\
& \left.+L_{m, 1}^{\wedge}(2 \pi n / N) \sum_{k=0}^{N-1} y_{k}^{(1)} w_{N}^{k n^{\prime}}\right) w_{\mu N}^{-n j} .
\end{aligned}
$$

This formula for the $r$ th derivative of the $N$-periodic Hermite spline interpolant is equivalent to the formula (9) for the $r$ th derivative of the cardinal Hermite spline interpolant. Thus, the values computed in Algorithm 4.1 can also be considered as approximate values of the $r$ th derivatives of the $N$-periodic Hermite spline interpolant.

\section{BIBLIOGRAPHY}

1. S. Lee and A. Sharma, Cardinal lacunary interpolation by g-splines I. The characteristic polynomials, J. Approx. Theory 16 (1976), 85-96.

2. P. R. Lipow and I. J. Schoenberg, Cardinal interpolation and spline functions III. Cardinal Hermite interpolation, Linear Algebra Appl. 6 (1975), 273-304. 
3. G. Meinardus and G. Merz, Hermite-Interpolation mit periodischen Spline-Funktionen, Numerical Methods of Approximation Theory (L. Collatz, G. Meinardus, and H. Werner, eds.), Birkhäuser, Basel, 1980, pp. 200-210.

4. G. Merz and W. Sippel, Zur Konstruktion periodischer Hermite-Interpolationssplines bei äquidistanter Knotenverteilung, J. Approx. Theory 54 (1988), 92-106.

5. G. Plonka, Periodic spline interpolation with shifted nodes, J. Approx. Theory 76 (1994), $1-20$.

6. An efficient algorithm for the periodic Hermite spline interpolation with shifted nodes, Numer. Algorithms 5 (1993), 51-62.

7. G. Plonka and M. Tasche, Efficient algorithms for periodic Hermite spline interpolation, Math. Comp. 58 (1992), 693-703.

8. M. Reimer, Cardinal Hermite-spline interpolation on the equidistant lattice, Numer. Math. 56 (1989), 345-357.

9. __ Zur reellen Darstellung periodischer Hermite-Spline-Interpolierender bei äquidistantem Gitter mit Knotenshift, Splines in Numerical Analysis (J. W. Schmidt and H. Späth, eds.), Akademie-Verlag, Berlin, 1989, pp. 125-134.

10. I. J. Schoenberg and A. Sharma, Cardinal interpolation and spline functions V. The B-splines for cardinal Hermite interpolation, Linear Algebra Appl. 7 (1973), 1-42.

Fachbereich Mathematik, Universität Rostock, D-18051 Rostock, Germany

E-mail address, G. Plonka: nfa016 @nfa.uni-rostock.d400.de

E-mail address, M. Tasche: tasche@mathematik.uni-rostock.d400.de 